\title{
Änderungen voraus
}

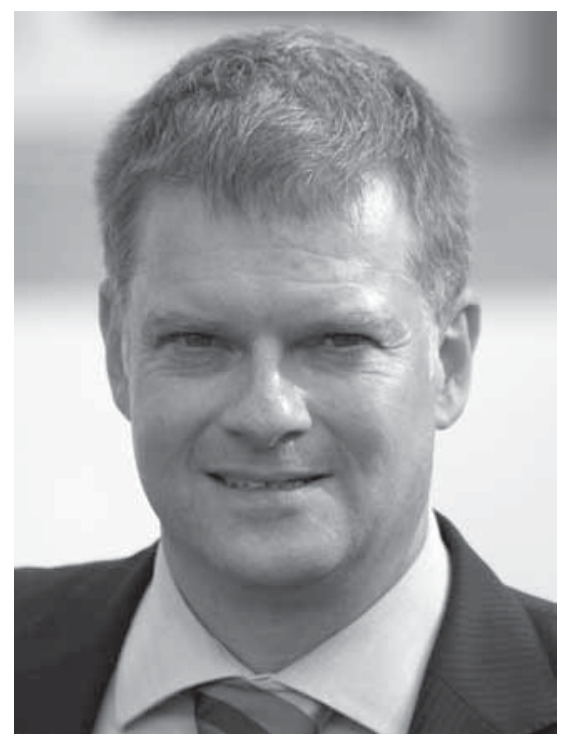

Das IT-Sicherheitsgesetz und die Datenschutz-Grundverordnung wirbeln derzeit viel Staub auf. Auch wenn auf die meisten Unternehmen zumindest hinsichtlich der datenschutzrechtlichen Anforderungen nur moderate Änderungen zukommen, sorgen die signifikant gestiegenen Sanktionen dafür, dass viele Verantwortliche sich fragen, was sie da wohl in Zukunft erwartet - und ob sie dafür ausreichend vorbereitet und gerüstet sind. Grund genug, einige der in diesem Zusammenhang relevanten, ganz praktisch-konkreten technischen und juristischen Fragestellungen einer näheren Betrachtung zu unterziehen.

Eine der Unsicherheit auslösenden Forderungen ist die nach dem „Stand der Technik" - einem unbestimmten Rechtsbegriff mit erheblichem Interpretationsspielraum. Michael Knopp geht diesem Begriff in seinem Beitrag auf den Grund und beleuchtet sowohl dessen Herkunft als auch die daraus abzuleitenden Erwartungen des Gesetzgebers.

Um der Forderung nach Nachweisen und Dokumentation sowohl im Datenschutz als auch bei der Informationssicherheit auf Dauer gerecht werden zu können, ist der Aufbau eines geeigneten Managementsystems unvermeidlich. Dass dies auch in einem sehr heterogenen und anspruchsvollen Umfeld gelingen kann, zeigt Dr. Ulrich Pordesch in seinem Erfahrungsbericht über die Einführung eines am ISO/IECStandard 27001 orientierten ISMS bei der Fraunhofer-Gesellschaft - und gibt zahlreiche Hinweise und Tipps für die Praxis.

Noch immer geistert die "disruptive Blockchain-Technologie" durch Leitartikel und Zukunftsvisionen. Mit den unterschiedlichen Facetten der Blockchain haben wir uns in Heft 8/2017, einem von Prof. Dr. Rüdiger Grimm und Prof. Dr. Andreas Heinemann inhaltlich gestalteten Schwerpunktheft intensiv auseinander gesetzt. Eine gründliche Beschäftigung mit diesem Thema hinterlässt unvermeidlich den Eindruck, dass die Erwartungen an die Blockchain gelegentlich die Möglichkeiten dieser Technik deutlich überstrapazieren. Einen wichtigen weiteren Aspekt, der in den aktuellen Diskussionen immer wieder übersehen wird, ergänzen Dr. Dirk Achenbach, Dr. Ingmar Baumgart und Jochen Rill in diesem Heft: Sie widmen sich den spezifischen Sicherheitsannahmen und -mechanismen, die der Blockchain zu Grunde liegen - und deren spezifische Eigenarten den Nutzen der Blockchain für zahlreiche der bisher vorgeschlagenen und diskutierten weiteren Anwendungen neben Zahlungssystemen zumindest in Frage stellen.

Das Thema "Notruf" hat uns bereits in mehreren Ausgaben der DuD beschäftigt $(11 / 2014,2 / 2016,5 / 2016,11 / 2016)$. Wer die Diskussion verfolgt hat, wird wissen, dass die Forderung , ,"moderne" Kommunikationsmedien endlich ", notruffähig" zu machen, auf erhebliche technische Hürden stößt: Schließlich muss das genutzte Netz eine hinreichend genaue, zuverlässige und fälschungssichere Lokalisierung eines Notrufs ermöglichen. Bastian Dreyer und Gerhard Kramarz-von Kohout stellen in ihrem Beitrag die aktuellen rechtlichen (EU-Regulierung) und technischen (VoLTE Notruf) Entwicklungen vor.

Schließlich beleuchtet Björn Voitel mit Blick auf die DS-GVO eine wahrscheinlich zukünftig häufiger gestellte Frage: Eignen sich Hash-Verfahren zur Anonymisierung personenbezogener Daten?

\section{Dirk Fox}

